\title{
Raperos y biógrafos: narrativas biográficas y educación para la paz con menores infractores ${ }^{1}$
}

\author{
Rappers and biographers: biographical narratives and \\ education for peace with juvenile offenders \\ Rappers e biógrafos: narrativas biográficas e \\ educação para a paz com jovens infratores
}

Recepción: 10/10/2019

Andrés Argüello Parra ${ }^{2}$

Universidad Pedagógica y Tecnológica de Colombia Grupo de Investigación HISULA - UPTC https://orcid.org/0000-0003-1181-028X

Sebastián Rojas Cepeda ${ }^{3}$ Centro Juvenil Amigoniano sede Tunja, Colombia Grupo de Investigación HISULA - UPTC https://orcid.org/0000-0003-3698-0277

Jeidy Cruz Guzmán ${ }^{4}$ Universidad Pedagógica y Tecnológica de Colombia Grupo de Investigación HISULA - UPTC https://orcid.org/0000-0003-1957-3151

Evaluación: 11/05/2020

Aceptación: 28/05/2020

Artículo de Investigación

https://doi.org/10.19053/01227238.11924

\section{RESUMEN}

El objetivo del artículo es analizar narrativas experienciales en el marco de una propuesta didáctica de Educación para la paz denominada "Mi autobiografía", realizada con una población del Sistema de Responsabilidad Penal Adolescente (SRPA) del Centro Juvenil Amigoniano de Tunja, Boyacá (Colombia). Originalidad: La investigación se sustenta en el principio de la historicidad del aprendizaje

$1 \quad$ El artículo es resultado de la investigación “Pedagogías, paz y poblaciones resilientes (2016-2019)", perteneciente al Grupo Historia y Prospectiva de la Universidad Latinoamericana - HISULA, código SIG 2706, de la Universidad Pedagógica y Tecnológica de Colombia, UPTC.

2 Doctor en Pedagogía de la Universidad Nacional Autónoma de México, UNAM - University of Łódź (Polonia). Posdoctorado en Educación, Ciencias Sociales e Interculturalidad. Investigador del Grupo HISULA, docente de la Escuela de Ciencias Sociales, Facultad de Ciencias de la Educación, director del semillero de investigación Pedagogía, formación docente e interculturalidad, Universidad Pedagógica y Tecnológica de Colombia, UPTC. Correo electrónico: jaime.arguello@uptc.edu.co

3 Licenciado en Ciencias Sociales de la Universidad Pedagógica y Tecnológica de Colombia, UPTC. Miembro del semillero Pedagogía, formación docente e interculturalidad, Grupo HISULA, UPTC. Correo electrónico: jhoan.rojas@uptc.edu.co

4 Magíster en Educación de la Universidad Pedagógica y Tecnológica de Colombia, UPTC. Miembro del semillero Pedagogía, formación docente e interculturalidad, Grupo HISULA. Correo electrónico: jeidycruz@yahoo.com 
humano, permitiendo analizar el contexto socio-cultural y los factores asociados que dan sentido desde el arte biográfico a las prácticas de vida de jóvenes infractores. Método: se examina una propuesta didáctica, basada en el enfoque socioeducativo de las historias de vida como práctica de formación, la cual se desarrolló en tres fases metodológicas, con las siguientes estrategias: sensibilización, diseño y producción de contenidos personales o autobiografías que fueron articuladas a estilos musicales del tipo Rap/Hip Hop, cine foro y visitas de artistas urbanos. Conclusión: La producción de textos inéditos fue estructurada y grabada como narrativas musicales, emergiendo categorías asociadas a las condiciones contextuales y dinámicas de vida subyacentes a la conducta delictuosa, que permiten concluir el aporte autobiográfico en la didáctica, expresado en el lenguaje de la música como potencial de reconducir las trayectorias delictivas hacia estadios de integración social, ciudadanías y construcción activa de paz.

Palabras clave: Menores infractores; educación en prisión; cultura de paz; estudios biográficos.
The objective of the article is to analyze experiential narratives within the framework of a didactic proposal for Peace Education named "My autobiography", carried out with a population of the Adolescent Criminal Responsibility System (SRPA) of the Amigoniano Youth Center of Tunja, Boyacá (Colombia). Originality: The research is focused on the principle of human learning historicity, allowing the socio-cultural context analysis and the associated factors that give meaning from biographical art to the life practices of young offenders. Method: a didactic proposal is examined based on the socio-educational approach of life stories as a training practice, which was developed in three methodological phases, with the following strategies:

\section{BSTRACT}

awareness raising, design and production of personal content or autobiographies that were articulated to Rap/Hip Hop style of music, forum cinema and visits by urban artists. Analysis: The production of unpublished texts was structured and recorded as musical narratives emerging categories associated with the contextual and dynamic conditions of life underlying criminal behavior, it allowed to conclude the autobiographical contribution in didactics expressed in the language of music, as a potential to redirect criminal trajectories towards stages of social integration, citizenship and active peace building.

Key words: Juvenile offenders; prison education; peace culture; biographical studies.

\section{RESUMO}

$\mathrm{O}$ artigo pretende analisar as narrativas vivenciais no âmbito de uma proposta didática de Educação para a Paz denominada "Minha Autobiografia", realizada com uma população do Sistema de Responsabilidade Penal do Adolescente (SRPA) do Centro Juvenil Amigoniano de Tunja, Boyacá (Colômbia). A pesquisa baseia-se no princípio da historicidade da aprendizagem humana, permitindo-nos analisar o contexto socio- cultural e as motivações que dão sentido às práticas de vida. A proposta didática, baseada na abordagem sócio-educativa das histórias de vida como prática formativa, foi desenvolvida em três fases metodológicas, nomeadamente: sensibilização, desenho e produção de conteúdos pessoais (autobiografias), que foram articulados com estilos musicais preferenciais dos jovens, fórum de cinema e visitas de artistas urbanos, entre outras 
estratégias. A produção de textos inéditos foi finalmente estruturada e gravada como produções musicais Rap/Hip Hop. Devido ao seu caráter narrativo integral, os trabalhos finais chamados: "La vida no es un cuento", "Improvisando", "Tomando conciencia" e "Autobiografía" foram revelados, onde foram encontradas categorias associadas às condições contextuais e dinâmicas da vida pessoal e social que definem a atividade criminal (grupo social, drogas, abandono familiar, etc.); construção de cenários de reflexividade, que conduzem a uma consciência dos danos causados e das próprias perdas; e um sentido de auto-confrontação, expresso na linguagem da música como potencial para redirigir as trajetórias criminais para etapas de integração social, nova cidadania e construção ativa da paz. Palavras-chave: Delinquentes juvenis; educação na prisão; cultura da paz; pesquisa biográfica.

\section{INTRODUCCIÓN}

El Código de Infancia y Adolescencia (Ley 1098 de 2006) define el Sistema de Responsabilidad Penal para Adolescentes (SRPA) como el "conjunto de principios, normas, procedimientos, autoridades judiciales especializadas y entes administrativos que rigen o intervienen en la investigación y juzgamiento de delitos cometidos por adolescentes de catorce (14) a dieciocho (18) años al momento de cometer un hecho punible" (art. 139). Las sanciones contempladas por el SRPA, que plantean una "finalidad protectora, educativa y restaurativa", abarcan la privación de la libertad que se cumple en un Centro de Atención Especializada.

Dentro de la función educativa ofertada en dichos centros, se encuentra la realización de los ciclos de educación básica y media que el menor cumple como parte de las actividades tendientes a mejorar su reintegración a la sociedad.
La presente investigación surge de una práctica pedagógica realizada durante el semestre académico 2018II, en el desarrollo de la asignatura de Ciencias Sociales, en el Centro Juvenil Amigoniano de Tunja (Boyacá), uno de los Centros de Atención Especializada en Colombia ${ }^{5}$, cuyo énfasis fue el diseño, ejecución y evaluación de una propuesta de Educación para la Paz con menores infractores. En efecto, la institución requería integrar a sus dinámicas curriculares establecidas los lineamientos de la Ley 1732 de 2014 para explicitar la cultura de paz como parte de la educación básica y media en un escenario de posconflicto.

Ello supuso el planteamiento de un problema didáctico $y$, en un sentido más amplio, de un problema educativo debido a las connotaciones ético-sociales derivadas del campo. En efecto, ¿cómo pensar un escenario de formación de paz con quienes han sido, precisamente, sujetos activos

\footnotetext{
5 En Colombia, un Centro de Atención Especializada es la entidad encargada de dar cumplimiento a una sanción privativa de libertad ordenada por la autoridad judicial hacia un menor de edad declarado penalmente responsable por alguna acción delictiva establecida en el sistema legal vigente, en concordancia con el SRPA.
} 
propiciadores de violencias? ¿Cómo lograr al menos un nivel básico de representatividad y pertinencia en el intento de aportar a la formación pos-delictiva de adolescentes sentenciados por distintas conductas punibles? ¿Qué tipo de diseños pedagógicos serían viables en un entorno donde las actividades de tipo académico generan predominantemente resistencia, repulsión y apatía?

Para ir tejiendo pistas a partir de tales interrogantes, los investigadores efectuamos un análisis de contexto basado en amplias jornadas de observación de las dinámicas escolares y organizacionales, así como diálogos con las distintas personas que cumplían funciones de asesoría, acompañamiento y vigilancia en el Centro, para elaborar algunas pautas tendientes al cumplimiento de la Cátedra de Paz en un escenario con características únicas.

En la revisión del estado del conocimiento, los estudios previos sobre la intervención con menores infractores desde la perspectiva de la Educación para la paz destacan, comúnmente, un modelo de justicia restaurativa (Hurtado, 2013) y la práctica de sus estrategias intrínsecas, tales como la mediación víctima-ofensor, conferencias de grupos familiares, círculos de discusión o sentencia y mesas comunitarias de reparación ${ }^{6}$. Así, el proceso educativo se entiende integrado a la recuperación del sujeto jurídico, esto es, a la responsabilidad del individuo ante la ley y ante la comunidad civil, en la cual se inscriben sus conductas. La educación para la paz está aunada a la educación democrática y a la formación del ciudadano.

El enfoque de Educación para la paz como intervención restaurativa privilegia los derechos de las víctimas a la verdad, a la justicia, a la reparación y a la no repetición, superando la mirada estrictamente punitiva; es decir, la paz se entiende como el aseguramiento de los derechos. En ese sentido de asociar la restauración de los derechos con la pedagogía se registra la monografía titulada "Procesos de justicia restaurativa utilizados en el ámbito pedagógico de menores infractores pertenecientes al sistema de Responsabilidad penal del Centro de Internamiento Preventivo Amigoniano (CIPA)", realizada por Justine Daniela Farfán Ballesteros en la Universidad Distrital Francisco José de Caldas en 2016. Una de las conclusiones sintetiza bien la perspectiva de estudio que comprende de manera específica la educación restaurativa en las instituciones amigonianas:

Gracias a las modificaciones que ha sufrido la legislación colombiana en materia de atención al menor infractor se tiene un cambio en la concepción y tratamiento al mismo, lo que permite que de un paradigma de atención tutelar se constituya uno de protección integral, donde se considera al joven como un sujeto tanto de derechos como de deberes, que al momento de cometer un acto delictivo debe ser sometido a medidas que permitan su formación integral y garanticen el cumplimiento de sus

6 Diana Britto Ruiz, Justicia Restaurativa. Reflexiones sobre la experiencia de Colombia (Loja, Ecuador: Editorial de la Universidad Técnica Particular de Loja, 2010). 
derechos humanos, a través de los cuales se busque la reparación tanto del ofensor, la víctima y la comunidad. ${ }^{7}$

Así mismo, como parte de la revisión de antecedentes, resultó muy significativo el análisis de la experiencia "El diario de los escritores libres" realizada por la maestra Erin Gruwell ${ }^{8}$ en la escuela secundaria Woodrow Wilson, de Long Beach, California (EE. UU.), y que fue llevada al cine por el director Richard LaGravenese con el título Freedom writers. En esta experiencia pedagógica se aprecia la imposibilidad de la maestra Gruwell para ejercer la docencia con población de estudiantes en riesgo por su trasegar delictivo, pandillerismo y tensiones raciales, situaciones que hacen de la escuela una extensión de las violencias cotidianas, hasta que implementa progresivamente una técnica de formación basada en diarios, en el relato de las historias, en el ejercicio de escribir como acto de (re) conocimiento para dar palabra al dolor; una apuesta que concluye en un lento - a veces desalentador, pero eficaz - proceso de empoderamiento y dignidad en los conflictivos estudiantes.

Reconociendo en esta y en otras prácticas pedagógicas el potencial de las historias de vida para llevar a cabo estrategias de formación desde las propias vivencias, experiencias y anécdotas, particularmente cuando se trata de poblaciones en especial condición de vulnerabilidad, se definió la dimensión biográfica como un eje central en la implementación curricular de los ciclos de ciencias sociales dedicados a la cultura de la paz.

Esta opción epistemológica permitiría construir dinámicas conducentes a revelar los propósitos de los estudiantes como personas y agentes constructores de no-violencia, partiendo de las trayectorias constitutivas de sus historias de vida: el conocimiento de sí, la historia social, las expectativas de futuro, sus intenciones de reparación y no repetición de infracciones; en suma, una interpretación personal y colectiva de las implicaciones sociales de la paz basada en la propia historicidad.

Se propone, entonces, la "Cátedra de la Paz" como un espacio de comprensión social desde la historia biográfica, donde el adolescente cuenta con las herramientas teóricas, didácticas y de expresión artística para fomentar un pensamiento reflexivo útil en el proceso rehabilitador, para sí mismo y para su entorno, tomando en cuenta la realidad de las poblaciones juveniles que sufren directamente las problemáticas de la violencia en Colombia.

El presente artículo se estructura desde los elementos básicos de la investigación: referentes conceptuales, metodología, resultados y discusión; y, por último, conclusiones. Como se verá a continuación, en los distintos apartados se desarrollan los fundamentos teóricos, metodológicos e interpretativos de la estrategia pedagógica denominada "Mi autobiografía" como propuesta de una

7 Justine D. Farfán Ballesteros, "Procesos de justicia restaurativa utilizados en el ámbito pedagógico de menores infractores pertenecientes al sistema de Responsabilidad penal del Centro de Internamiento Preventivo Amigoniano (CIPA)" (monografía para la licenciatura en Biología, Universidad Distrital Francisco José de Caldas, 2016), 84.

8 Erin Gruwell, El diario de los escritores de la libertad. Su historia. Sus palabras (Barcelona: Elipsis Ediciones, 2007). 
Educación para la paz realizada con menores infractores en el Centro Juvenil Amigoniano.

\section{Educación para la paz y violencias}

Los referentes conceptuales de la presente investigación destacan, principalmente, dos orientaciones: la producción de la violencia como ethos de una nación; y, frente a ella, la educación para la paz como mandato social y político. Hay que partir recordando que en un país como Colombia - que produce víctimas en cada esquina de sus ciudades y en cada hectárea de sus campos-, la violencia es un fenómeno endémico y estructural basado en procesos de acción coercitiva y sistemática del poder establecido contra grupos sociales y personas históricamente vulnerabilizadas mediante el sostenimiento, explícito o tácito, de distintas condiciones como la miseria, la pobreza multidimensional, el desempleo, la desigualdad de oportunidades, las acciones militares contra civiles, la criminalización de la protesta social, la ineficacia de la justicia y la estigmatización de la diversidad sociocultural, entre muchas otras. Así lo recuerdan Almada y Cortés al decir: "Un primer nivel de conceptualización que es necesario precisar es el de la violencia en su forma estructural. Esta contiene un determinante político por lo que se ha definido como 'una acción de coerción, de ejercicio del poder que se da en el contexto de relaciones entre personas o grupos sociales, cuando la distribución de poder (económico, político, físico, simbólico) es asimétrica o desigual'"'.

De esta manera, se ha configurado una organización social que no solo es proclive a la producción de violencias en sus múltiples manifestaciones, sino al sostenimiento de los poderes que las amparan; un verdadero ethos nacional sostenido por el analfabetismo político y la amnesia histórica como basamentos de los problemas más determinantes del país, que se vienen heredando generacionalmente - durante décadas - al punto que ningún ciudadano hoy, ni joven ni viejo, podría declarar haber vivido algún tiempo de bonanza social o ausencia plena de las guerras.

Es claro que el fenómeno de los menores delincuentes no es exclusivo de un país como Colombia; sin embargo, la niñez que se desarrolla en el marco de una "violencia atmosférica", encuentra todas las condiciones para alimentar dicho sistema de exclusión, muerte y contravención de los valores fundantes de una sociedad (Cruz, 2007). Sin eufemismos justificatorios, los menores infractores son producto de este sistema, ya naturalizado en la cultura nacional colombiana, que los ha instrumentalizado para los más abyectos propósitos de la guerra, el crimen y toda suerte de ilegalidad.

La llamada sociedad disciplinaria, constituida sobre la hegemonía del poder y del saber, produce sus propios monstruos, de los cuales espera salir indemne.

9 María Teresa Almada y Miguel Cortés. Jóvenes: ¿Violencia o convivencia? Técnicas participativas para la resolución noviolenta de conflictos. (Ciudad Juárez: Universidad Autónoma de Ciudad Juárez, 2013), 16. 
Crea, así, una suerte de sociofagia de concepciones, axiologías y mediaciones simbólicas que devienen en sistemas de necrofilia, necesarios para sostener su propia hegemonización del poder ${ }^{10}$. En esa medida, el Estado ha forjado una escuela normalizadora, disciplinaria y de control que no cumple con su papel histórico de transformar vidas para construir un plano civilizatorio basado en el respeto a la vida, a las diferencias culturales y al trabajo honesto, sino que reproduce el marco de conservación de las identidades canónicas, aunque tácitamente estén asociadas a las formas delictivas del poder.

No en vano, los factores de riesgo en la producción de menores delincuentes se intensifican en el marco de los sectores populares y marginales que, desde sus contextos de origen, son influenciados y, de cierta manera, adoctrinados por la "cultura traqueta"11 como ideal de vida y de realización que crea procesos alternativos de identidad personal y social ante la crisis de la familia tradicional, de la escuela y, en general, de las instituciones con funciones educadoras en el tejido social. Los menores infractores no son generación espontánea de la inevitabilidad del mal; además de las estructuras socio-políticas que los producen, nacen y se crían en contextos específicos de la ruptura familiar patriarcal y heteronormativa. La familia, como un organismo histórico, dinámico y con variantes regionales, para el caso colombiano, es un inevitable factor de la conformación social, por la ya clásica idea de definirse como célula de la sociedad ${ }^{12}$.

En tanto los sistemas educativos, inmersos en las lógicas empresariales de calidad, productividad y resultados, no logran abordar los desafíos derivados de semejante estado de descomposición humana y social que deja la violencia estructural, se establece el desafío de incorporar una educación para la paz como proyecto contracultural que explicite los valores alternativos a la guerra y a todos los insumos que la hacen posible.

A este propósito, la iniciativa del Estado colombiano se ha acentuado desde una perspectiva de posconflicto que intenta comprender el rol de la escuela como agente constructor de una cultura de paz:

El Estado, entendiendo la escuela como un escenario de reproducción social, promulgó, en el 2014, la Ley 1732 desde la cual se busca promover la "Cátedra

10 Comentando a Foucault, Reinaldo Giraldo explica: "En Vigilar y castigar, el pensador francés entra en el ámbito carcelario para estudiar el surgimiento y la extensión de las formas de disciplina en cuanto que tecnologías políticas y procedimientos disciplinarios que no anulan la individualidad, sino que la producen a través de una estrategia de normalización caracterizada por el encierro. Este encierro comprende una gran variedad de formas y de métodos para la corrección que van desde los trabajos forzados y el aislamiento hasta la educación. Busca someter a los desviados a la normalización y a la vigilancia". Reinaldo Giraldo Díaz, "Prisión y sociedad disciplinaria", Entramado vol. 4, n. ${ }^{\circ} 1$ (2008): 83.

11 Renán Vega, “La formación de una cultura 'traqueta' en Colombia”, Revista CEPA vol. 1, n. 18 (2014).

12 La desaparición paulatina de la familia tradicional y la asunción de modelos emergentes de tipo monoparental, extensa, polimorfa, etc., se ha nutrido con el fenómeno de la transición de una sociedad rural a una de mayoría urbana, configurando una formación social residente en las periferias de las ciudades que genera situaciones como anomia moral y desarraigo cultural coadyuvantes de los grandes problemas sociales nacionales tales como la exacerbación del conflicto en las ciudades, la marginalidad y, junto a ello, la producción de menores infractores: "Con los cambios de las tipologías familiares, se han producido desaparición de familias tradicionales, en parte por las rupturas, divorcios, separaciones, etc.; los hijos quedan a cargo de uno de los progenitores que en ocasiones mantienen mala relación con su ex-pareja y otro progenitor, lo que permite a los hijos vivir con menores controles y mayor desatención, lo que podría llevarles a entrar en círculos de riesgo generando causas de riesgo y conductas disruptivas debido a la 
para la paz" en todas las instituciones educativas del país. Posteriormente, en el 2015 publicó el decreto 1038 por el cual se reglamenta dicha Cátedra al interior de las instituciones educativas. De acuerdo con este decreto, el objetivo primordial de la Cátedra para la Paz es "contribuir al aprendizaje, la reflexión y al diálogo sobre [...] cultura de la paz, educación para la paz y desarrollo sostenible" (Decreto 1038,2015, p. 1). ${ }^{13}$

Empero, la educación para la paz en Colombia, antes de ser un mandato legal es un mandato ético que, si bien integra un nivel formativo en torno a los contenidos históricos, políticos, sociales y culturales de la violencia estructural, conlleva necesariamente un énfasis transformador de las condiciones posibilitantes de toda barbarie e inhumanidad. Por ello, la educación para la paz no es un simple tema de índole instruccional o asignaturista, sino un problema de formación integral humana para un sistema civilizatorio alternativo. Se trata de

[...] la necesidad de construir una cultura basada en la negociación de significados para posibilitar un pluralismo humanista y la reconstrucción de un nuevo orden social. Por ello, la educación desde una perspectiva crítica e intercultural juega un papel central para redefinir las subjetividades y el compromiso con un proyecto ético que contribuya a la emergencia de una narrativa contra-hegemónica del concepto canónico de paz. ${ }^{14}$

El concepto de paz que subyace a esta orientación educativa se basa en la pluralización de su semántica, esto es, la idea de paces desarrollada por el filósofo Vicent Martínez Guzmán ${ }^{15}$, que supera el enfoque liberal moderno centrado en los derechos individuales y reconoce las múltiples formas de construcción de paz que se edifican desde la diversidad en la producción de subjetividades y en las prácticas intersubjetivas: "Lo cotidiano, y no lo universal, debe servir de punto de partida para el fomento de culturas de paces, propiciando espacios y lugares de enunciación propios y endógenos para enfrentar la mercantilización de la paz en el modelo neoliberal"16. Así, frente a las distintas expresiones de la violencia, que desbordan el nivel macrosocial y se instalan en la particularidad de la experiencia humana, es necesario acudir a una comprensión plural del relato antagónico de las violencias. Las paces se inscriben como un ejercicio de construcción continua de las identidades, de las relaciones y de los valores

desorganización familiar, con síndrome de padres ausentes". Concepción Nieto-Morales, Nuria Cordero y María Josefa Vásquez-Fernández, coords. Intervención con colectivos desfavorecidos: Descendiendo a la práctica de la intervención profesional. Madrid: Editorial Dyckinson (2017), 284.

13 Eduard Esteban Moreno, "Educación, conflicto y posconflicto en Colombia", Diálogos de saberes, n. ${ }^{\circ} 46$ (2017): 126.

14 Andrés Argüello y Anctil Priscyll, "From 'Polymorphism of Violence' to the Complexities of Peace: Towards an Integral Dignity in Colombia”, en Human Dignity. Establishing Worth and Seeking Solutions, eds. Edward Sieh y Judy McGregor (Londres: Palgrave Macmillan, 2017), 240-241.

15 Vicent Martínez Guzmán, Filosofía para hacer las paces (Barcelona: Icaria editorial, 2009).

16 Priscyll Anctil Avoine y Nadia Johanna Paredes Saavedra, "Pensar la construcción de paces en Colombia: la (ir)relevancia teórico-práctica de la decolonialidad”, Análisis vol. 50, n. 93 (2018): 324. 
humanos dominantes desde el ser en situación, desde la condición biografiable de la vulneración o de la pérdida.

Dicho proyecto concibe la educación como una tarea social basada en la valoración ética de la alteridad capaz de conjurar la reproducción de causas generadoras de delito e ilegalidad en los menores. En los alcances de esta finalidad, la educación para la paz requiere de diseños didácticos creativos, provocadores de reflexividad y compromiso, y un especial sentido de trans/escolaridad que abarque los distintos escenarios sociales desde una mirada de formación moral, política y ciudadana. En efecto, “el enfoque pedagógico tiene diferentes niveles de actuación. Uno de ellos es favorecer, precisamente, climas más humanizadores donde los sujetos se sientan reconocidos en su identidad y aspiraciones" ${ }^{\prime 17}$.

Plantear la educación para la paz en un escenario de reclusión de menores infractores supone un complejo proceso de acercamiento a las realidades individuales para relevar las configuraciones de personalidad que pueden influir en patrones de disociación social constituyentes de acciones delictivas. Ello requiere un enfoque pedagógico que potencialice las cualidades personales y permita la expresión de capacidades e inteligencias desde una presunción positiva de restauración y enmienda.

El educador, por su parte, en el marco específico de estos contextos retadores y contradictorios, singulariza su responsabilidad social y pedagógica al requerir estrategias de mediación desde el acompañamiento ponderado, la escucha activa y la comunicación empática, no tanto para discriminar, someter y disciplinar sino para ayudar a comprender la complejidad de las relaciones individuo-familia-escuela-sociedad en procura de una reconstrucción vital con profundo sentido de desarrollo integral humano.

Finalmente, este enfoque orgánico de educación ante condiciones de violencia estructural, con sus distintas implicaciones, podría sintetizarse como sigue:

En la educación para construir cultura de paz es importante tener en cuenta la dimensión cognitiva respecto del problema de violencia y conflictos, pero es más significativa la participación desde la visión multidimensional del ser humano, en la búsqueda y planteamiento de soluciones eficaces en ámbitos donde prime el respeto a los derechos humanos y al medio ambiente; demanda por tanto, la implementación de un enfoque humanístico que permita fundamentalmente el aprendizaje significativo, colaborativo y autónomo que desarrolle el pensamiento crítico y la ciudadanía activa y democrática en el estudiante, lo que le permitirá incluirse de manera sencilla y natural en la cultura para ser parte activa y permanente en la construcción de paz; mientras explora de manera directa y creativa los distintos escenarios, experiencias y proyectos a fin de obtener aprendizajes vivenciales y con sentido de la realidad de su entorno. ${ }^{18}$

17 Fernando Gil Cantero, "La acción pedagógica en las prisiones. Posibilidades y límites", revista española de pedagogía año 68, n. $^{\circ} 245$ (2010): 55

18 Isabel Hernández Arteaga, José A. Luna y Martha C. Cadena, “Cultura de paz: una construcción desde la educación”, Revista Historia de la Educación Latinoamericana vol. 19, n. ${ }^{\circ} 28$ (2017), 168. 
Tales criterios conceptuales han contorneado el diseño metodológico de esta investigación a partir de un marco cualitativo e interpretativo que destaque el papel central del sujeto en la valoración de sus trayectorias y en los distintos ámbitos de construcción sociocultural.

\section{Método}

El presente estudio se inscribe en el horizonte epistemológico de las historias de vida como prácticas de formación en el contexto de la educación de adultos, que configura el movimiento socioeducativo de las historias de vida en formación. Desde esta perspectiva, las indagaciones investigativas "se centran en las nociones de reflexividad, representaciones, sentido, creencias, valores... y se vuelve hacia la historicidad del sujeto y de los aprendizajes" ${ }^{\prime 19}$. Así, los diversos escenarios de desenvolvimiento de los procesos humanos junto a las formas discursivas del yo, se tornan configuradores de modos de ser que orientan los contenidos de la acción en lo social. De esta manera, las narrativas biográficas permiten "revelar los modos como se tejen los vínculos entre el sujeto y el mundo de las esferas sociales donde él vive e interactúa" ${ }^{20}$, donde se abarca el universo de las causalidades interpretativas - no necesariamente fácticas - que regulan las prácticas de vida y el complejo proceso de apropiación de la historia social como historia personal.

La investigación (auto)biográfica recoge las intencionalidades circundantes a la construcción de las identidades en registros de espacio-tiempo ligados a modos específicos de historicidad del sujeto: "Analiza cómo los individuos integran, estructuran, interpretan los espacios y las temporalidades de sus contextos histórico-culturales para examinar, por ese camino, el proceso de construcción del sujeto (o del grupo) en la interacción dialéctica entre el espacio social y el espacio personal mediante el(los) lenguaje(s)"21.

Para comprender cómo los individuos o los colectivos atribuyen sentido al devenir de la existencia, en el itinerario de la formación humana, de acuerdo con las características de la población participante de este estudio, resulta de particular interés señalar que la investigación (auto) biográfica es, de igual manera, un posicionamiento epistemopolítico al "considerar lo que la persona piensa sobre ella y sobre el mundo, el sentido que confiere a sus acciones y la toma de conciencia de su historicidad (...). El sujeto es agente y paciente de interacciones sociales" 22

En efecto, cuestionarse sobre la construcción permanente de sí mismo, además de expresar una idea asociada a la educación a lo largo de la vida, manifiesta un posicionamiento consciente con relación a las condiciones sociales y políticas

19 Maria da Conceição Passeggi, “A pesquisa (auto)biográfica: por uma hermenêutica descolonizadora”, Coisas do Gênero vol. 2 , n. ${ }^{\circ} 2$ (2016) 305. Cursivas y traducción propia.

20 Maria da Conceição Passeggi, “Aproximaciones teóricas a las perspectivas de la investigación (auto)biográfica en educación”, Educación y Pedagogía vol. 23, $\mathrm{n}^{\circ} 61$ (2011)26.

21 Ibid., 29.

22 Ibid., 30,32 
del entorno para la superación de la dualidad estructural entre el individuo y su socialidad. Esta dilucidación de la conciencia histórica, desde una mirada emancipadora y decolonizadora, "coloca en el centro del proceso la capacidad humana de reflexividad autobiográfica orientada a la acción del sujeto en la emancipación y empoderamiento (...) frente a interpretaciones culturales excluyentes" ${ }^{\prime 23}$.

Desde aquí, el andamiaje teórico-metodológico a partir de los principios comprehensivos de la investigación (auto)biográfica aplicados a la educación, se sustenta en la noción de biograficidad ${ }^{24}$, la cual releva la conciencia histórica de los aprendizajes, tal como se puede apreciar en el proceso de campo realizado con los menores del centro de atención especializada, que fue base de esta investigación.

\section{a) Contexto y unidad de análisis}

El estudio se desarrolló en el Centro de Atención Especializada o Centro Juvenil Amigoniano de la ciudad de Tunja, Boyacá, regentado por la Congregación de Religiosos Terciarios Capuchinos o Amigonianos, fundada en 1889 por fray Luis Amigó, la cual atiende escuelas de reforma o correccionales, centros para la educación de jóvenes en situación de riesgo o de conflicto que incluye recuperación de personas con problemas de dependencias. Entre los principios orientadores de la congregación se destacan, entre otros: la atención educativa personalizada, gradual y progresiva, que busca promover el desarrollo integral; y la convicción en las posibilidades de cada persona para superar las dificultades que va encontrando en su vida ${ }^{25}$.

En Tunja, Boyacá, el Centro Juvenil es una institución reconocida por la Secretaria de Educación Municipal con la resolución n. ${ }^{\circ} 0087$ de febrero del 2010. Su naturaleza es privada, de metodología presencial y se localiza en el barrio Libertador, al sur-occidente de la ciudad. El Centro de Atención Especializada Amigoniano cuenta con estudiantes que están vinculados al Sistema de Responsabilidad Penal para Adolescentes (SRPA).

El centro cuenta con cuatro secciones (casas), todas ubicadas de manera separada, que son lugar de residencia de los jóvenes. Cada casa cuenta con entre 25 y 30 jóvenes, distribuidos según la modalidad a la que está inscrita su población en el sistema penal (CAE/SIP). La denominación de las casas es Fortaleza, Nuevo amanecer, Trascender y Génesis.

Fortaleza: sección donde habita la mayoría de jóvenes en modalidad de Centro de Internamiento Preventivo, alrededor de veintidós, cuya sanción no supera los seis meses.

23 Maria da Conceição Passeggi, “A pesquisa (auto)biográfica: por uma hermenêutica descolonizadora”, Coisas do Gênero vol. 2, n.o 2 (2016) 308 .

24 Bettina Dausien, “'Aprendizaje biográfico’ y ‘biograficidad. Reflexiones para una idea y una práctica pedagógicas en la formación de personas adultas", Sinéctica. Revista Electrónica de Educación, n. ${ }^{\circ} 45$ (2015).

25 "Centro de Pensamiento, Doctrina y Pedagogía Amigoniano, CPA", www.amigonianoscg.org/cpa/ (2014). 
Nuevo amanecer: sección ocupada, en su mayoría, por reclusos en modalidad de Centro de Atención Especializada, cuya sanción es a largo plazo; esta sección es la más compleja en el sistema de resocialización ya que acoge a jóvenes con dificultades de asimilación al proceso; alberga alrededor de veinticinco jóvenes.

Trascender: sección con mayoría de jóvenes también en modalidad de Centro de Atención Especializada, con sanciones a largo plazo, pero que asumen actitudes más propositivas al cumplimiento del proceso y a su desarrollo académico secundario que, pronto a culminar, genera oportunidades de estudio técnico o profesional. Recibe treinta jóvenes en promedio.

Génesis: sección cuyos jóvenes están en la última cúspide del proceso de resocialización, asimilaron su proceso y tienen la disposición de cumplir en su totalidad con las normativas establecidas; ellos cuentan con el beneficio de poder empezar su proceso de educación técnica o profesional, con unos permisos otorgados por los entes institucionales (juez, ICBF, director del Centro) para salir en horarios establecidos en razón de estudio; obtienen y mantienen estos beneficios siempre y cuando cumplan estrictamente con la normativas internas. La sección cuenta con treinta jóvenes.

El escenario del presente estudio es la asignatura de Ciencias Sociales, orientada como Cátedra de la Paz, en los ciclos V y VI correspondientes a los niveles de educación media (décimo y undécimo), actividad académica cursada por los menores como parte de su programa de formación integral. El curso de ambos ciclos contaba con 17 jóvenes, en su mayoría integrantes de las secciones Trascender y Nuevo amanecer. El ejercicio de escritura autobiográfica se dirigió a la totalidad de la clase, pero según los criterios de representatividad y pertinencia, fueron seleccionados finalmente cuatro participantes, todos ellos varones, entre 19 y 21 años, procedentes de Sogamoso (Boyacá), Pacho (Cundinamarca), Quibdó (Chocó) y Tunja, con sanciones de internamiento entre los ocho y los veinticuatro meses.

\section{b) Procedimiento metodológico}

Durante el desarrollo de la asignatura se llevaron a cabo tres fases: sensibilización, diseño y producción de contenidos personales relacionados con los contenidos sociales del plan de trabajo de la asignatura. En primer lugar, la sensibilización se entiende como una etapa de acercamiento de los participantes a las nociones orientadoras de la Educación para la paz desde un enfoque de narrativa personal, de manera que pueda contribuirse a la generación de sentido escolar en la articulación de estilos artísticos musicales de interés para cada joven.

Para tal fin, se suscitaron mesas de discusión de las temáticas a tratar con base en la proyección de dos materiales fílmicos: Voces inocentes, dirigida por Luis Mandoki, que retrata la incursión de menores de edad en la guerra civil salvadoreña con la infinidad de vicisitudes que ello representa; y, 80 millas, del director Curtis Hanson, en la cual un joven de escasos recursos (Eminem) sueña 
con ser cantante pero encuentra todas las contrariedades a su alrededor para cumplir ese ideal de vida; no obstante, en la consecución de su empeño, él va transfiriendo la propia historia de vida en la escritura para expresarla luego en la música, llegando a ser uno de los raperos más importantes del mundo.

Considerando las historias reales que sustentan estos materiales fílmicos, la actividad del cine-foro pretendió ambientar procesos de auto-identificación para generar en los menores un incentivo a la escritura autobiográfica donde la expresión artística permitiera recoger las distintas dimensiones de su proyecto de vida en el marco de condiciones personales, sociales y culturales marcadas por la violencia y el delito.

De igual manera, como parte de la sensibilización se invitaron a la clase expertos en juventud, ritmos urbanos y construcción de paz, destacando la actividad con un rapero colombiano, Mc. Shadow, quien durante su proceso de llegar a ser cantante vivió situaciones de violencia, drogas, armas y criminalidad, pero que en la misma música empezó a reconstruir su proyecto de vida y se enfocó en utilizar el Hip Hop como herramienta de socialización para transmitir un mensaje de vida, en especial a jóvenes en situaciones similares de vulnerabilidad. El testimonio específico de este rapero invitado al curso expresa que, a pesar de afrontar múltiples situaciones de violencia o drogadicción, la regeneración de la existencia puede ser posible, ilustrando además un papel protagónico de la creación musical en la reflexividad personal y en el impacto social.

Posteriormente, en la fase de diseño se llevó a cabo la escritura de materiales autobiográficos. Según se ha comentado en los fundamentos del enfoque, la estrategia pedagógica denominada aquí "Mi autobiografía", admite distintas dinámicas para la creación y consolidación de espacios que incentivan el aprendizaje reflexivo y el diálogo sobre la cultura de la paz. Dentro de las distintas posibilidades se privilegia en el presente trabajo el uso de las narrativas personales como insumo de expresiones artísticas musicales de género Hip-Hop/Rap, donde los jóvenes del centro de internamiento cuentan con especial afinidad y talento para realizar variadas composiciones. 
Foto 1. Taller de escritura biográfica

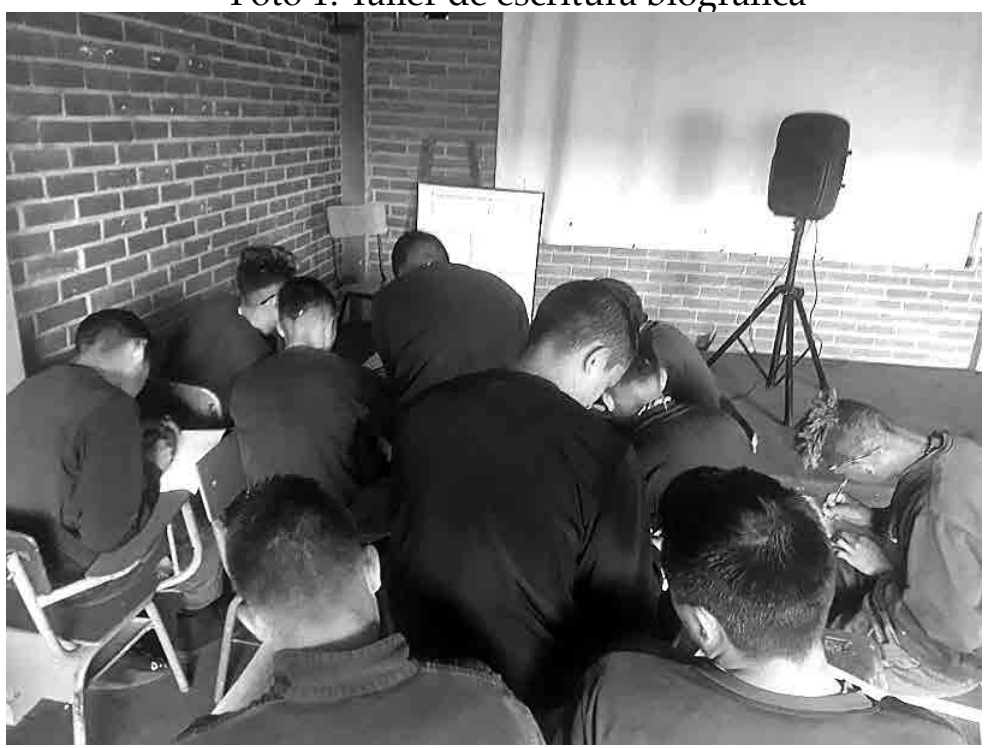

Fuente: Archivo de Sebastián Rojas Cepeda.

Desde estas estrategias de ambientación se incentivó el ejercicio del “Diario autobiográfico" mediante la cual los jóvenes realizarían la escritura de momentos representativos de su vida, a partir de ciertos lineamientos orientadores que sirvieran de anticipo a la expresión oral y a la musicalización de las historias. Se explicó previamente que tales lineamientos funcionarían solo como una pauta para la narración y no como un conjunto de preguntas cerradas tipo encuesta o cuestionario.

Las pautas de contenido se sintetizan en tres momentos entresacados del objeto de estudio aquí referenciado: infancia u orígenes, donde se indaga por los recuerdos de niñez, las condiciones que rodearon los primeros años, cómo vivieron la infancia, con quiénes, bajo qué contextos o situaciones características. El segundo momento, de tipo valorativo, se concentra en dos preguntas orientadoras: ¿Qué quisiera vivir nuevamente? y ¿Qué no quisiera haber vivido?, como una forma de ponderar las tensiones entre la conveniencia del pasado y la ocurrencia de hechos punitivos. Por último, el tercer momento, de carácter proyectivo, pregunta por el papel del joven dentro de la sociedad, la visualización personal en un futuro próximo y las concepciones construidas en torno a algunos conceptos representativos e identitarios tales como cultura, familia, amistad, educación, religión y justicia.

Inicialmente, la confección del diario se realizó en mesas de escritura tutoriadas, es decir, sesiones de clase dedicadas a la construcción de dicha narrativa con acompañamiento del docente o equipo asesor, implementando distintas estrategias didácticas que permitieran a los estudiantes desplegar sus múltiples talentos. En un momento posterior, luego de percibir alguna afinidad con la 
actividad escritural, se concedió el uso libre de los diarios para ser diligenciados en momentos extra-clase, de acuerdo con las dinámicas del Centro y el involucramiento de los adolescentes a lo largo de la investigación.

Foto 2. Diarios autobiográficos

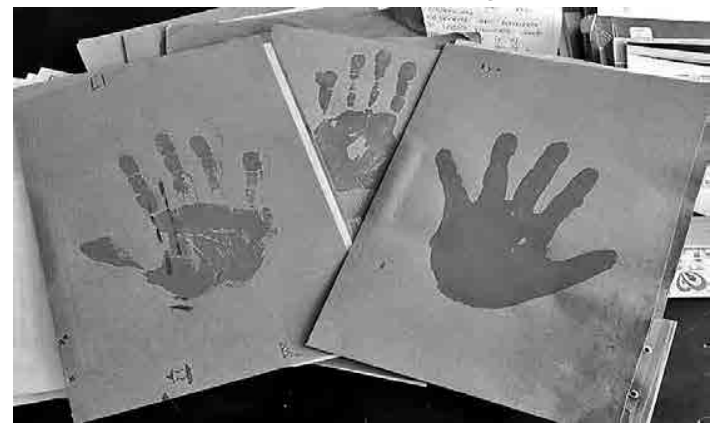

Fuente: Archivo de Sebastián Rojas Cepeda.

El cierre del ejercicio formativo, o fase de producción, comenzó por una revisión general de los textos y, posteriormente, un perfilamiento de quienes mejor podrían asumir el diseño de la musicalización por cuanto, si bien la actividad de producir narrativas autobiográficas se dirigió a todo el curso de ciclo V y VI, era claro que no todos tenían el mismo empeño, disposición y simpatía para la realización del trabajo.

Estructurar canciones propias, realizar modificaciones en la conformación de los textos y preparar la grabación de los discos, fue la etapa siguiente a la revisión general de los trabajos. Por medio de la música buscamos que los jóvenes se motivaran a contar su experiencia de vida, teniendo como fin producir una historia de reconocimiento transformativo sobre la base de distintas fuentes teóricas de las ciencias sociales y de autoconocimiento como aporte a la construcción de paz. 


\section{Foto 3. Grabación de composiciones}

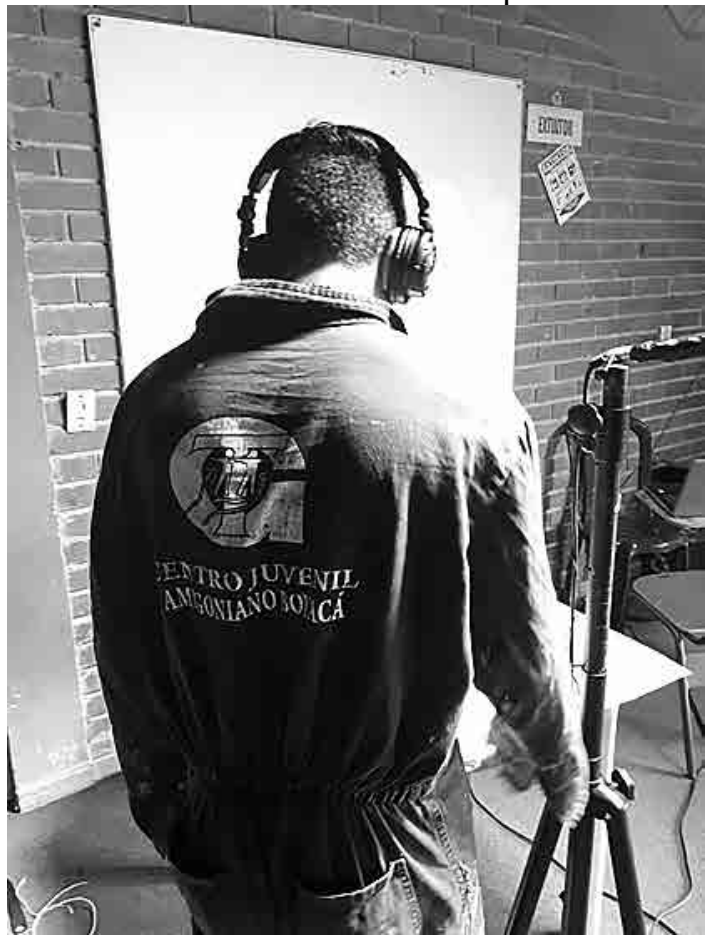

Fuente: Archivo de Sebastián Rojas Cepeda.

Se relevaron los trabajos finales de cuatro participantes, Sneyder, Jefferson, Ernest y Robert ${ }^{26}$, tanto por el carácter integral de sus narrativas como por la estructuración de las mismas en canciones y ritmos orientados a los talleres musicales desde los ejemplos prácticos de la escena local del Rap, constituyendo, asimismo, una motivación a los demás compañeros sobre la finalidad de plasmar retóricas personales con intención divulgativa. Gran parte de los estudiantes se mostraron receptivos al proceso de socialización, reproducción y grabación de sus canciones, efectuado, además, de manera artesanal desde los recursos con los que cuenta el Centro.

\section{Resultados y análisis}

Dentro de las cuatro autobiografías construidas por los estudiantes en reclusión, se destacan ciertas dinámicas de la vida personal y social que se pueden recoger en dos categorías dominantes: la escritura narrativa biográfica como reflexividad para la autoconciencia, en el marco de la producción lírica; y, junto a ese acto de reconocimiento, el sentido de enmienda y de reparación basada

26 Nombres modificados para proteger la identidad de los menores. 
en la compunción por los daños ocasionados a sí mismos, a sus familias y a las víctimas.

Los dos ejes categoriales emergen de la producción de informaciones en los trabajos finales, denominados: La vida no es un cuento, Improvisando, Tomando conciencia y Autobiografía. Para llevar a cabo el análisis de estos "documentos de vida" desde un enfoque hermenéutico-crítico que sustenta el método biográfico integrativo-experiencial, se siguieron tres fases ${ }^{27}$ :

Fase 1: Escrutinio de materiales o fase documental. Se refiere, básicamente, al reconocimiento formal de los materiales recolectados, cotejar su legalidad - entendida como la pertinencia frente a los ejes estructuradores del estudioy elaborar una clasificación preliminar de hallazgos a partir de estructuras de contenido o segmentos temáticos.

Fase 2: Generación de categorías de análisis (o emergentes), o fase comprensiva. Corresponde a un momento de abstracción analítica sobre los hallazgos del escrutinio formal. Para ello se determinaron esquemas de dominancia, relación y yuxtaposición advertidas en la actividad de biografización de los participantes para establecer jerarquizaciones sobre la base de agrupamientos de categorías recurrentes o puntos de capitoneado.

Fase 3: Establecimiento de representaciones o fase textual. Finalmente, sobre la base relacional de las fases precedentes, se construyó una versión entrecruzada de relatos, como se muestra a continuación, en diálogo con los objetivos de la investigación y sus posibles incidencias. Valga resaltar que en esta etapa del análisis se destacan los contenidos narrativos en su producción natural, ofreciendo una versión articulada de los mismos para plantear una comprensión integradora en torno al fenómeno estudiado desde los dos ejes categoriales definidos.

Así, en primer lugar, al plantear la reflexividad para la autoconciencia, se aprecian las nociones de contexto que perfilan el quehacer delictivo. Queda evidenciado, por ejemplo, el marco de drogadicción y la fuerza del grupo social de amigos que domina el ambiente en el que se desarrolla el joven:

Empecé con el consumo ya no veía nada, me dejé cegar solo por el humo y mis viejos notaron malos cambios, las notas ahora son las de un vago (...) empecé a reemplazar los abrazos, los besos que le daba a mis papás por el trago y los amigos (La vida no es un cuento).

Este aspecto se encuentra desde el ya clásico trabajo de Clifford R. Shaw planteado en el marco de la naciente Escuela de Chicago, en 1930, The Jack-Roller: A delinquent boy's own story, el cual recoge la autobiografía de Stanley - un joven

27 Para el desarrollo de las fases de análisis en la investigación biográfica educativa se sigue el trabajo de Andrés Argüello Parra, Educación, biografía y derechos humanos. Un estudio de Rodolfo Stavenhagen, itinerante de alteridades (Bucaramanga: USTA-IEM, 2014). 
de amplia trayectoria delincuencial - sobre la base de sus experiencias, influencias y valoraciones socioculturales.

Para Echarri, el concepto de adolescentes en exclusión social se define por las características especiales y conductas de riesgo proclives a la disrupción del individuo frente al canon legal de la sociedad, condiciones que resulta indispensable abordar en el sistema educativo:

Si algo caracteriza comúnmente al colectivo de jóvenes en exclusión social es el poseer acusados factores de riesgo. Normalmente presentan una historia de crecimiento y desarrollo personal producida en contextos o escenarios de precariedad (económica, en muchos casos, y social, en la práctica totalidad de los mismos). ${ }^{28}$

Algunos de esos factores de riesgo proclives a la constitución de subjetividades cifradas por la criminalidad, son ponderadas como sigue:

La precocidad de los primeros delitos contra la ley (robos, venta de drogas, etc.) es un indicador clave en la predicción de una delincuencia grave en la adolescencia. Resultados académicos débiles, abandono escolar, supervisión parental pobre, asociación con pares delincuentes, consumo abusivo de drogas y alcohol, ocio y ausencia de ocupaciones pro sociales para organizar los tiempos libres, la valoración del estilo de vida delictual son algunos de los elementos que van a contribuir al aumento y la agravación de la actividad delictual (delitos criminales) en la adolescencia e incluso en la persistencia de esta delincuencia en la edad adulta. Esos factores de riesgo van a consolidar la trayectoria delictual en el adolescente, aún más intensamente si se completa con un proceso de construcción de identidad personal, marginal y delictual. ${ }^{29}$

De esta forma, el restablecimiento identitario impelido por la fractura social aparece vinculado a nuevos marcos de referencia colectiva, casi siempre, en el ámbito de grupos de pares reflejo que cumplen funciones de afianzamiento de las propias visiones disruptivas: "En estos casos los adolescentes suelen buscar refugio en sus iguales, buscando amigos de su mismo perfil. De este modo, el grupo modela y refuerza socialmente sus conductas de riesgo (actividades predelictivas o delictivas, consumos de drogas, etc.), que refuerzan nuevamente su situación de conflicto social".$^{30}$

En el ejercicio de reflexividad, el adolecente reconoce la influencia del grupo social en su proceder errático, y al mismo tiempo destaca la ruptura que supone la deslealtad de su círculo de pares:

28 Fernando Echarri, Metodología educativa para adolescentes en exclusión social (Navarra: Ediciones Universidad de Navarra, 2017), 25 .

29 Jacques Dionne y Alba Zambrano, "Intervención con adolescentes infractores de ley”, en El desafío de la intervención psicosocial en Chile. Aportes desde la psicoeducación, eds. María Beatriz Vizcarra y Jacques Dionne (Santiago de Chile: RIL editores, 2008), 250-251.

30 Echarri, Metodología educativa, 23. 
sin escrúpulos tus socios te venderán porque ellos no pensarán y en la jaula te encerrarán y ni un pan te comprarán porque solo el pensar de ellos es querer soplar (Improvisando).

Aparece, así, el sentimiento de abandono, de frustración y de pérdida que no solo es derivado del espacio social de la acción delictiva en sí misma sino de la fractura de esas vinculaciones afectivas que parecían perdurables:

Los errores de la vida se pagan tarde o temprano y tus supuestos amigos no te brindan ni una mano, cuando estabas en la calle te tratan como a un hermano, pero cuando estás adentro te tratan como un marrano (Tomando conciencia).

O, también, al entonar:

yo estoy demente rafagando liricas como un delincuente, hoy me encuentro aquí encerrado porque sé que en muchas cosas yo he fallado, pero en la noche cuando sueño, mi conciencia he reflexionado, mi corazón está pesado, pero en nadie yo he pensado, la vida se va tan rápido de lo que nada un pescado (Improvisando).

De la admisión del error surge la conciencia de daño y pérdida, donde la constatación del vacío es la consecuencia más clara e incluso apabullante de la práctica delictiva. Sin familia y sin amigos, la soledad se convierte en escenario de afrontamiento de la propia identidad y, en últimas, en el efecto punitivo más determinante:

hoy prefiero a mi familia que en la noche ir a farrear, yo extraño muchas cosas que pensé yo no llegaría a perder (Improvisando).

En efecto, la decepción de confianza de la familia, de los seres queridos más íntimos, será uno de los quicios articuladores de la reflexividad como toma de conciencia y, así mismo, como restablecimiento de la vida:

escucha madre lo lamento, perdón madre mía, por aquellas desveladas, las lágrimas derramadas y yo en vez de consolarte nunca te decía nada (Tomando conciencia)

Aún más, en un caso particular, emerge también el sentimiento de paternidad:

es una necesidad esta gran caída para valorar lo que afuera yo tenía, el amor de mi vida, la luz de mis días, lo que le da un gran sentido a mi vida... mi hija (Autobiografía).

Por ello, además del acto de reconocimiento de la culpa, se constata un sentido de redefinición de la vida, de enmienda y reparación a partir de un sólido compromiso fundado en los vínculos humanos de familia: 
Cada día voy $\mathrm{pa}^{\prime}$ delante, no me friego soy valiente, por mi madre lo que sea así me tilden de delincuente, de los errores se aprende, siempre sigo sonriente vivo con la frente en alto porque soy sobreviviente, encerrado, encausado y un poco desorientado mostrándole a la gente que se puede y que he cambiado, a pesar de lo que hice, el daño yo he reparado (Tomando conciencia).

O, dicho desde la experiencia de otro narrador:

él solo labró su destino, ahora sí se arrepiente, ahora sí le duele el menosprecio que tenía hacia sus padres cada día, el remordimiento por hacerle daño a quien no debía, pero ellos siempre perdonan y son los que nunca abandonan (La vida no es un cuento).

Para él mismo, es necesario que ese afecto sea transformador:

ahora lamento haberme alejado de los seres que yo amo, retroceder el tiempo es lo que he añorado para no tener solo recuerdos sino vivir el momento, la demora es salir de aquí para darle todo a todos los que me hicieron existir y nuevamente estar con ellos llenándome de sentimientos bellos, ahora solo queda esperar que termine mi proceso para irme en libertad (La vida no es un cuento).

La reintegración en la sociedad se basa en el principio de la relación empática, que es una estructura vincular esencial previamente fracturada por la comisión del delito. En la perspectiva del delincuente no hay capacidad para apreciar la condición de alteridad; por eso la víctima solo es un instrumento de la magnificación del yo que se impone desde patrones consolidados de violencia y coacción. De hecho, la violencia de todo tipo es un sistema de disposiciones egotistas.

De ahí que la intervención socioeducativa en menores con medidas judiciales se ordena a la recuperación de la convención social, tanto en clave ética como legal, con base en los postulados de la pedagogía social, donde la familia, como escenario de relaciones afectivas estructurantes, es el primer eslabón de socialidad.

Por último, el análisis de los relatos autobiográficos realizados en el marco del presente estudio muestran cómo la educación para la paz, en el caso específico de los menores infractores, se traduce en nuevas directrices de existencia que explicitan valores proclives a la convivencia, a la disrupción del delito y a la construcción de un proyecto de vida con sentido humano, tal como lo apreciamos en los siguientes fragmentos:

pronto afuera en la calle de nuevo para un nuevo inicio, como no (...) valora cada día que pasas en la libertad y después que no la tengas no vayas a lamentar el no haberla aprovechado por cada día malgastado, será peor aún cuanto te encuentres encerrado (Tomando conciencia). 
De igual forma, en La vida no es un cuento:

acá es donde tú no vales nada aquí tu no mandas ninguna banda, pero tu reflexionas y te das cuenta que no todo es droga (...) la vida no es un cuento aprovecha los momentos porque tarde o temprano se convierten en recuerdos (La vida no es un cuento).

El mismo sentido aparece en el narrador de Autobiografía:

Es caer y levantarse para demostrar lo que los demás piensan que no vas a alcanzar, cumplir mis sueños y trabajar para el buen sustento de mi familia, nada más (Autobiografía).

La enmienda contiene una intención restaurativa, la cual expresa una decidida voluntad de cambio, que estaría asociada a los procesos de intervención educativa propiciados por el Centro, la cual contiene diversidad de experiencias no solo de formación académica sino de desarrollo de habilidades para el trabajo, de favorecimiento de valores humanos y espirituales, de acompañamiento psicosocial permanente y de proyección restaurativa a un futuro próximo forjado en novedad de vida.

El castigo es propiciador de la conciencia ética de sí; es decir, el surgimiento del sujeto reflexivo aparece ligado a la escena de la sanción social. El papel restaurador del Centro puede leerse en esa clave, tal como menciona Butler comentando a Nietzsche: "La institución del castigo me ata a mi acto, y cuando se me castiga por haber cometido tal o cual hecho, surjo como un sujeto de conciencia y, por consiguiente, como un sujeto que reflexiona, de alguna manera sobre sí mismo"31.

\section{CONCLUSIONES}

Dentro de los distintos criterios conceptuales derivados de esta investigación pedagógica, se podrían destacar aquellos que establecen articulaciones entre el proceso de aula para el caso de las ciencias sociales, la educación para la paz y la utilización de narrativas biográficas con poblaciones en condición de especial vulnerabilidad.

En primer lugar, si bien el contexto social cumple una función generadora en la producción de perfilamientos criminales, también alberga un potencial preventivo, donde cobra razón de ser el papel de la educación. Por ello, un principio a tener en cuenta es la confluencia de factores no solo en el ámbito de la causalidad de la conducta punible sino en las intervenciones socioeducativas preventivas, protectoras y restaurativas.

31 Judith Butler, Dar cuenta de sí mismo. Violencia ética y responsabilidad (Buenos Aires: Amorrortu editores, 2009$), 28$. 
En este sentido, la educación con adolescentes infractores cumple una función de restablecimiento y armonización de las múltiples dimensiones del ser personal, convocando las fragmentaciones del individuo a un nuevo sentido de sí en la comunidad: "Se trata, en definitiva, de favorecer en estos jóvenes el desarrollo de recursos personales (habilidades sociales y de toma de decisiones, autoestima, autoeficacia, autocontrol emocional, capacidades, competencias profesionales, etc.) y recursos sociales (adultos positivos de referencia, ambiente estable, formación adaptada, acceso al empleo, etc.)"32.

Para ello, un aspecto de especial profundización es el desarrollo de la sociabilidad de los sujetos que, desde distintos mecanismos de intervención educativa, conduzca al fortalecimiento de la conciencia empática. En el caso de la educación para la paz, esta dimensión adquiere una importancia sustantiva al concebir al individuo no solo en su condición de vulnerabilidad, riesgo o conflicto social, sino como agente propiciador de acciones delictivas o vulneración de derechos en contra de personas y comunidades específicas.

La educación para la paz es fundamental en todos los ámbitos educativos $y$, particularmente, en el marco de pedagogías emergentes, al considerar la producción de subjetividades como desarrollo de los individuos en sus entornos para la gestión razonable de conflictos y la convivencia en la diversidad. Esta apuesta pedagógica cobra especial relevancia en escenarios de resocialización que conciben una visión positiva de ser humano, de cambio y renovación bajo los parámetros sociales establecidos.

En ese marco de formación para la paz desde la conciencia de sí, la reflexividad ofrece el potencial de reconducir las trayectorias delictivas hacia estadios de integración social y nuevas ciudadanías por cuanto llevan al individuo a un nivel inédito de confrontación y de valoración de las conductas:

\begin{abstract}
En el corazón de esta pedagogía, se da una gran importancia a la estimulación de las capacidades de reflexión del joven. Detenerse y reflexionar es el primer deber de todos los miembros de la comunidad - jóvenes y educadores. Actuar sobre los errores de pensamiento corriente o distorsiones cognitivas delictuales, es intervenir para contradecir uno de los factores de riesgo importante del mantenimiento de la delincuencia. Porque si un adolescente justifica sus acciones delictuales con respuestas automáticas para conservar un funcionamiento egocéntrico, o para proteger emociones no confortables, persiste en su estilo de vida delictual y evita el desequilibrio que le produciría la preocupación por el otro. ${ }^{33}$
\end{abstract}

La contrición moral y el deseo de enmienda están contorneados por el ejercicio reflexivo y la restauración de la sociabilidad. Para tal fin, las narrativas autobiográficas se proponen como un ejercicio didáctico experiencial, recapitulativo de los postulados centrales de la educación para la paz con menores infractores:

32 Echarri, Metodología educativa, 23

33 Dionne y Zambrano, "Intervención con adolescentes infractores", 262. 
"yo solo busco la calma de algunos escritores que no piensan en la fama sino en corregir errores" (Improvisando). Los jóvenes participantes de la actividad, en términos generales, demuestran conexión con el ejercicio biográfico y la expresión de sus sentimientos de una manera artística. Poder integrar sus historias a sus intereses musicales constituye no solo una forma de apropiación sino de reconocimiento, e incluso, de reparación.

Los procesos educativos de sujetos constructores de paz, como se han entendido en este estudio, articulan las potencialidades didácticas del arte en donde los jóvenes sean partícipes directos desde el despliegue de sus talentos y la generación de estrategias que incentiven a otros jóvenes a seguir fomentando los valores democráticos de la convivencia, el respeto y el cumplimiento responsable de la ley.

Aprender con base en la propia experiencia es una de las características permanentes de las historias de vida, pero más aún, en contextos de adolescentes en condiciones de exclusión social pues implica una revaloración de los propios sentidos ante el mundo en el cual se desarrolla la existencia. La actividad didáctica que aquí denominamos "Mi biografía”, demuestra ser conveniente y propositiva en el ejercicio pedagógico con jóvenes sancionados penalmente. En el caso particular, fue posible consolidar un espacio académico-artístico con los jóvenes de los ciclos V y VI, en donde, además de presentar las diferentes temáticas asociadas a la clase, se motivó un pensamiento de compromiso y transformación. En la relación sujeto-sujeto, se construye una relación afectiva con el tutor en términos de ganar el esquivo respeto y direccionalidad; los menores asumen su protagonismo dentro del proyecto, razonan los objetivos y se hacen partícipes de los resultados.

La música termina siendo un estímulo para contar la experiencia de vida, no tanto desde la vergüenza del infractor sino desde el aporte a la construcción de paz. El Hip Hop y el arte de hacer rimas es un reconocimiento de los lenguajes de los destinatarios en la acción educativa para superar progresivamente la visión panóptica determinista en torno al menor recluido: “Tenemos la oportunidad de reparar el daño que causamos (...). Todavía hay esperanza" (Tomando conciencia).

La Cátedra de la Paz en este escenario tiene una función de impacto en la comunidad pues anticipa el ideal de la transformación de vida y aporta a la reconstrucción del tejido social que viven muchos jóvenes propensos a cometer delitos. Pensar que los jóvenes infractores en la totalidad de los casos están condenados al delito significaría negar la experiencia de las transformaciones generadas por intervenciones educativas, derrotismo compartido con la cultura delictiva donde se ve la educación como algo que "no va a servir en la vida" o que simplemente "no interesa".

Desplegar talentos desde el contexto, con herramientas y dinámicas diferentes, resulta esencial para una acción pedagógica significativa. Es preciso, así, el diseño de espacios de discusión, debate, análisis y construcción colectiva, pero también de creación y autoconocimiento, de lenguajes y posibilidades como incentivos para entender la paz en cuanto anhelo colectivo, concientiza- 
ción del proyecto humano y civilización de la alteridad, según se expresa en los contenidos de sus canciones, ansias de nueva vida, no repetición de los errores cometidos, oportunidad para la compasión y la justicia.

\section{REFERENCIAS}

Almada, María Teresa y Miguel Cortés. Jóvenes: ¿Violencia o convivencia?: Técnicas participativas para la resolución noviolenta de conflictos. Ciudad Juárez: Universidad Autónoma de Ciudad Juárez. 2013.

Anctil Avoine, Priscyll y Nadia Johanna Paredes Saavedra. "Pensar la construcción de paces en Colombia: la (ir)relevancia teórico-práctica de la decolonialidad". Análisis 50, n. 93 (2018): 317-335.

Argüello Parra, Andrés. Educación, biografía y derechos humanos. Un estudio de Rodolfo Stavenhagen, itinerante de alteridades. Bucaramanga: USTA-IEM, 2014.

Argüello Parra, Andrés y Priscyll Anctil. “From 'Polymorphism of Violence' to the Complexities of Peace: Towards an Integral Dignity in Colombia". En Human Dignity. Establishing Worth and Seeking Solutions, editado por Edward Sieh y Judy McGregor. Londres: Palgrave Macmillan, 2017, 231-245.

Britto Ruiz, Diana. Justicia Restaurativa. Reflexiones sobre la experiencia de Colombia. Loja (Ecuador): Editorial de la Universidad Técnica Particular de Loja, 2010.

Butler, Judith. Dar cuenta de sí mismo. Violencia ética y responsabilidad. Buenos Aires: Amorrortu editores, 2009.

"Centro de Pensamiento, Doctrina y Pedagogía Amigoniano", CPA. Disponible en: www.amigonianoscg.org/cpa/ (2014).

Cruz y Cruz, Elba. "El concepto de menores infractores". Revista del Posgrado en Derecho de la UNAM 3, n. 5 (2007): 335-355.

Dausien, Bettina. “'Aprendizaje biográfico' y 'biograficidad'. Reflexiones para una idea y una práctica pedagógicas en la formación de personas adultas". Sinéctica. Revista Electrónica de Educación, n. ${ }^{\circ} 45$ (2015): 1-16. http://www.scielo.org.mx/pdf/sine/n45/n45a5.pdf

Dionne, Jacques y Alba Zambrano, "Intervención con adolescentes infractores de ley". En El desafío de la intervención psicosocial en Chile. Aportes desde la psicoeducación, editado por María Beatriz Vizcarra y Jacques Dionne. Santiago de Chile: RIL editores, 2008, 250-251.

Echarri Iribarren, Fernando. Metodología educativa para adolescentes en exclusión social. Navarra: Ediciones Universidad de Navarra, 2017.

Gil Cantero, Fernando. "La acción pedagógica en las prisiones. Posibilidades y límites". Revista española de pedagogía 68, n. ${ }^{\circ} 245$ (2010): 49-64. Disponible en: https://dialnet.unirioja.es/ descarga/articulo/3099332.pdf

Giraldo Díaz, Reinaldo. "Prisión y sociedad disciplinaria". Entramado 4, n.o 1 (2008): 82-96. Disponible en: https://revistas.unilibre.edu.co/index.php/entramado/article/download/3332/2724

Gruwell, Erin, El diario de los escritores de la libertad. Su historia. Sus palabras. Barcelona: Elipsis Ediciones, 2007.

Hernández Arteaga, Isabel, José A. Luna y Martha C. Cadena. “Cultura de paz: una construcción desde la educación". Revista Historia de la Educación Latinoamericana 19, n. 28 (2017): 149-172. https://doi.org/10.19053/01227238.5596

Hurtado Rodríguez, Hernando. “Justicia juvenil: entre el proteccionismo de Estado y la garantía de derechos". Cuadernos de Trabajo Social26, n. ${ }^{\circ} 1$ (2013): 171-180.

Instituto Colombiano de Bienestar Familiar. Acceso el 19 de septiembre de 2019. https://www.icbf. gov.co/bienestar/proteccion/responsabilidad-penal

Jiménez Marín, Dinnora. "Responsabilidad penal juvenil en Colombia: de la ideología tutelar a la protección integral". Diálogos de Derecho y Política, n. ${ }^{\circ} 1$ (2009): 133-151. Disponible en https://revistas.udea.edu.co/index.php/derypol/article/view/2103

Martínez Guzmán, Vicent. Filosofía para hacer las paces. Barcelona: Icaria editorial, 2009. 
Moreno, Eduard Esteban. "Educación, conflicto y posconflicto en Colombia”. Diálogos de saberes, n. 46 (2017): 125-142. Disponible en: https://dialnet.unirioja.es/descarga/articulo/6260867.pdf

Nieto-Morales, Concepción, Nuria Cordero y María Josefa Vázquez-Fernández, coordinadoras. Intervención con colectivos desfavorecidos: descendiendo a la práctica de la intervención profesional. Madrid: Editorial Dykinson,, 2017.

Passeggi, Maria da Conceição. "A pesquisa (auto)biográfica: por uma hermenêutica descolonizadora". Coisas do Gênero 2, n. 2 (2016): 302-314. Disponible en: http://ism.edu.br/periodicos/ index.php/genero/article/view/2918/2718

Passeggi, Maria da Conceição. “Aproximaciones teóricas a las perspectivas de la investigación (auto)biográfica en educación". Educación y Pedagogía 23, n. ${ }^{\circ} 61$ (2011): 25-40. Disponible en: https://dialnet.unirioja.es/descarga/articulo/4159459.pdf

Vega, Renán. "La formación de una cultura 'traqueta' en Colombia". Revista CEPA 1, n. 18 (2014). Disponible en: http://www.rebelion.org/noticias/2014/2/180935.pdf

\section{Cómo citar:}

Argüello Parra, Andrés; Rojas Cepeda, Sebastián; Cruz Guzmán, Jeidy. "Raperos y biógrafos: narrativas biográficas y educación para la paz con menores infractores". Revista Historia de la Educación Latinoamericana. 22 No. 35 (2020): 119-143 https://doi.org/10.19053/01227238.11924

(c) $(1) \Theta($ @) Esta obra está bajo una licencia Creative Commons. Reconocimiento-No Comercial-Sin Obra Derivada 2.5 Colombia. 
